\title{
Association of night eating habits with metabolic syndrome and its components: a longitudinal study
}

\author{
Junko Yoshida ${ }^{1,2}$, Eri Eguchi $^{1 *}$, Kenjiro Nagaoka ${ }^{1}$, Tatsuo Ito ${ }^{1}$ and Keiki Ogino ${ }^{1}$
}

\begin{abstract}
Background: Night time eating is a risk factor for metabolic syndrome and obesity. The aim of this study was to investigate whether dinner immediately before bed, snacks after dinner, or combinations of both were associated with metabolic syndrome and its components in a large Japanese cohort.

Methods: We enrolled 8153 adults aged 40-54 years who participated in specific medical checkups in an Okayama facility from 2009 to 2010 and from 2013 to 2014. Age-adjusted and multivariable-adjusted odds ratios of metabolic syndrome and its components in participants with both night eating habits for an average of 3.9 years were evaluated. The relative excess risk due to interaction (RERI) was utilized to determine the supra-additive interaction of both eating habits on metabolic syndrome and its components.

Results: The multivariable-adjusted odds ratio for obesity for those with both eating habits compared to those with neither habit was 2.11 (95\% confidence interval [Cl], 1.42-3.15) for men and 3.02 (95\%Cl, 1.72-5.29) for women. Both habits had a supra-additive interaction effect on obesity development in women (RERI, 1.67; RERI\%, 85.0; $p=0.058$ ), although this result was not significant. In women, there was an association between eating habits at night and metabolic syndrome, but in men it was unrelated. Both night eating habits were associated with dyslipidemia in men and women.

Conclusions: These findings suggest the need for intervention and awareness among individuals with night eating habits to mitigate further complications.
\end{abstract}

Keywords: Night eating, Metabolic syndrome, Obesity, Abdominal obesity, Dyslipidemia

\section{Background}

Obesity and metabolic syndrome are associated with cardiovascular diseases, including cerebrovascular disease and ischemic heart disease, as well as type 2 diabetes mellitus [1-4]. The National Health and Nutrition Survey 2015 showed that $54.0 \%$ of men and $18.4 \%$ of women aged 40-74 years in Japan were strongly suspected of having metabolic or borderline metabolic syndrome, which are important health concerns in Japan because of the large financial burden associated with metabolic syndrome [5]. A medical checkup that specifically targets metabolic syndrome was introduced in April 2008 to prevent lifestyle-

\footnotetext{
* Correspondence: eri_eguchi@okayama-u.ac.jp

${ }^{1}$ Department of Public Health, Okayama University Graduate School of Medicine, Dentistry and Pharmaceutical Sciences, 2-5-1 Shikatacho, Kita-ku, Okayama 700-8558, Japan

Full list of author information is available at the end of the article
}

related diseases through early screening. People at risk of metabolic syndrome received specific health guidance by public health nurses and registered dietitians, and they reported improved examination values after three years. Medical expenses and lifestyle-related disease rates also decreased.

Eating habits are directly related to obesity. For example, eating until full and eating quickly can cause unhealthy weight gain [6], and skipping breakfast affects both waist circumference and body mass index (BMI) [7]. In a 4-week trial including a total of 36 obese men and women, breakfast skippers showed an increase in serum total cholesterol [8]. In CARDIA (Coronary Artery Risk Development in Young Adults), daily breakfast eaters also had a lower risk of hypertension (HR, 0.74; 95\% CI, 0.63-0.86) and metabolic syndrome (HR, 0.63; 
95\%CI, 0.54-0.75) than infrequent breakfast eaters $(0-3$ times a week) [9]. Night time eating, in particular, has been identified as a risk factor for metabolic syndrome and obesity. An intervention study of healthy adults in the United States reported that calorie consumption after $8 \mathrm{PM}$ was positively correlated with $\mathrm{BMI}$ and revealed that calorie consumption after $8 \mathrm{PM}$ was an independent predictor of high BMI after adjustments for age, sleep duration, and sleep timing [10]. Late-night eating was associated with an OR for obesity of $1.62(95 \% \mathrm{CI}$, 1.10-2.39) compared with no late-night eating among 3610 Swedish men and women [11]. A cross-sectional study among 239 US adults reported that individuals who consumed $\geq 33 \%$ of their total energy intake (TEI) in the evening had twice the risk of being obese (OR, 2.00 ; 95\% CI, 1.03-3.89) compared with individuals who consumed $<33 \%$ of their TEI at night [12]. Soga et al. studied 4912 individuals in their 30s who participated in health checkups for young adults and showed that the multivariable-adjusted odds ratio (OR) for metabolic syndrome was approximately twice as high in women who had dinner immediately before bed than in those who did not [13]. Additionally, the combination of latenight eating and skipping breakfast was associated with a greater risk of metabolic syndrome among Japanese adults ( $n=60,800$; age, $20-75$ years) [14]. Another intervention study of fourteen healthy subjects in the United States suggested that eating during the biological night time might increase the risk of weight gain and obesity. Subjects lived for $\sim 6$ days to simulate a daytime work schedule followed by a 3-day night shift schedule. The total daily energy expenditure decreased by $\sim 3 \%$ on each of the two night shift days, which consisted of day time sleep followed by afternoon and night time wakefulness [15].

Previous large-scale studies examining night eating habits in Japanese population have reported varying results. Soga et al. reported that among 4912 individual, $43.4 \%$ males and $15.2 \%$ females had a tendency of "dinner immediately before bed", and $26.0 \%$ males and 23.2\% females had a tendency for "snacks after dinner" [13]. Ashizawa.et al., on the other hand, analyzed 278,989 people in Chiba prefecture in Japan and reported that $16.2 \%$ males and $9.0 \%$ females had a tendency for "dinner immediately before bed", and 5.9\% males and $6.9 \%$ females had a tendency for "snacks after dinner" [16]. According to Overview of national medical expenses in 2016, diseases related to metabolic syndrome formed $37.8 \%$ of all medical expenses [17], suggesting the need for further investigation into this disease. To our knowledge, no large-scale populationbased studies assessing the additive effect of both night eating habits ("dinner immediately before bed" and "snacks after dinner") on the prevalence of metabolic syndrome and its components have been conducted in Japan.

In the present study, we assessed whether the presence of both night eating habits was associated with metabolic syndrome and its components, such as obesity, abdominal obesity, dyslipidemia, impaired blood pressure, and impaired blood glucose.

\section{Methods \\ Subjects}

The participants of this study were 17,534 workers and their spouses aged 40-55 years who underwent medical checkups, with a particular focus on metabolic syndrome, at a facility in Okayama between 2009 and 2010 (baseline). Of these, 9198 workers and their spouses participated in the medical follow-up checkups (between 2013 and 2014; 52.5\%). We excluded individuals with missing data on "dinner immediately before bed" and "snacks after dinner" $(n=1045)$. Data of 8153 individuals (4875 men and 3278 women) were used in the final analyses (follow-up rate $46.5 \%$ ). This study protocol was approved by the ethics committee of Okayama University (approval number: 1032) and conducted in accordance with the Declaration of Helsinki. Considering the longitudinal nature of the study and large number of participants, verbal or written consent was not obtained from all participants. The study was advertised on posters in medical examination centers for a certain period of time. Patients were asked to participate either through the website or in person. Their participation in the survey was taken as consent to participate. This protocol was approved by the ethics committee. The data were analyzed anonymously.

\section{Measurements}

Body mass index (BMI), waist circumference, blood pressure, and metabolites were measured once a year during the annual medical check-up at the Junpukai Health Maintenance Center.

Height and body weight were measured, and BMI was calculated as weight $(\mathrm{kg}) /$ height $\left(\mathrm{m}^{2}\right)$. Based on the obesity criteria from the World Health Organization [18], obesity is defined as a BMI $\geq 25 \mathrm{~kg} / \mathrm{m}^{2}$. Abdominal obesity was defined as a waist circumference at the navel of $\geq 90$ $\mathrm{cm}$ for men and $\geq 80 \mathrm{~cm}$ for women. Blood pressure was measured twice in the sitting position using an automated blood pressure monitor (UDEX-TWIN, Well up).

Blood tests were conducted in a fasting state for the majority of subjects and in a non-fasting state for some subjects. Therefore, fasting blood glucose levels were not available for all subjects. In this study, glycated hemoglobin (HbA1c) levels were monitored and set at $\geq 5.6 \%$, which is the cut-off value defined for providing specific health guidance in Japan [19]. HbA1c levels were measured using 
high-performance liquid chromatography. High-density lipoprotein (HDL) and low-density lipoprotein (LDL) cholesterol levels were measured using an enzymatic method (direct method), and triglyceride levels were measured using an enzymatic-based colorimetric method.

Based on the information by IDF [20], we defined metabolic syndrome as any case that showed a waist circumference at the navel of $\geq 90 \mathrm{~cm}$ for men and $\geq 80 \mathrm{~cm}$ for women in addition to exhibiting $\geq 2$ of the following components: 1) dyslipidemia, with triglyceride level $\geq 150$ $\mathrm{mg} / \mathrm{dL}, 2)$ dyslipidemia, with HDL cholesterol level $<40$ $\mathrm{mg} / \mathrm{dL}$ for men and $<50 \mathrm{mg} / \mathrm{dL}$ for women, 3) hypertension with systolic blood pressure $\geq 130 \mathrm{mmHg}$ and/or diastolic blood pressure $\geq 85 \mathrm{mmHg}$, and 4) hyperglycemia, with HbA1c $\geq 5.6 \%$ [National Glycohemoglobin Standardization Program]. Participants receiving medications for dyslipidemia, hypertension, or hyperglycemia were considered to have the respective condition. Japan Diabetes Society HbA1c values were converted to the National Glycohemoglobin Standardization Program HbA1c values using the officially certified formula: HbA1c $($ NGSP $)(\%)=1.02 \times$ JDS $(\%)+0.25 \%$.

\section{Questionnaire survey}

We used the self-administered "standard questionnaire" [19] to assess the participants' dietary habits. Health care providers in Japan have used this questionnaire since 2008 to evaluate patients for metabolic syndrome. The questionnaire was developed based on the conventional National Health and Nutrition Examination Survey and questions specified by the Industrial Safety and Health Act $[19,21]$. It included selection/stratification questions (1-3 [medication use], 4-6 [medical history, present illness], and 8 [smoking history]), which are essential for the specific medical checkup [19]. It also included 22 other items such as frequency of exercise, weight gain, dietary habits, alcohol consumption, and sleeping habits. The participants answered "yes" or "no" to questions concerning night eating habits and other lifestyle-related behaviors. The questionnaire items are standard and have been widely used, and previous studies have showed that these lifestyle behaviors are related to the prevalence of metabolic syndrome [13, 16, 22].

\section{Night eating habits}

Night eating habits were defined as "dinner immediately before bed" (dinner within 2 hours of bedtime $\geq 3$ times/week) and "snacks after dinner" (snacks after dinner $\geq 3$ times/week).

\section{Other lifestyle-related behaviors}

Other lifestyle-related behaviors included smoking (current smoking), exercise ("I have engaged in light $\geq 30$-minute exercise more than twice/week for $\geq 1$ year"), physical activity ("I go for a walk or perform an equivalent physical activity for $\geq 1$ hour/day"), skipping breakfast ("I skip breakfast $\geq 3$ times/week"), and alcohol consumption (current alcohol consumption: " $<2$ go $[180 \mathrm{~mL} /$ go]" or " $\geq 2 g o$ ", where " $g o$ " is a traditional Japanese unit of volume measurement, corresponding to $23 \mathrm{~g}$ of ethanol). Alcohol consumption of $\geq 2$ go was defined as "excessive drinking".

\section{Statistical analysis}

The age-adjusted means and proportion of BMI, waist circumference, blood pressure, metabolite levels, metabolic syndrome, and lifestyle-related behaviors (smoking, exercise, physical activity, skipping breakfast, and alcohol consumption) at baseline (2009-2010) were calculated on the basis of the presence or absence of "dinner immediately before bed" and "snacks after dinner" and were compared using analysis of covariance (Dunnett's multiple comparison test). We compared the groups with one or both eating habits against a group without both habits.

Thereafter, the mean values of the BMI, waist circumference, blood pressure, and metabolite levels compared between baseline (2009-2010) and follow-up (20132014) using the Wilcoxon signed rank-tests. The prevalence of metabolic syndrome, night eating habits, smoking, physical activity, and alcohol consumption were compared using the McNemar's test.

Age- and multivariable-adjusted ORs for metabolic syndrome and its components were calculated using logistic regression analysis in 7287 participants (4262 men and 3025 women), after excluding 866 participants with metabolic syndrome, at baseline to assess the relationships between both eating habits at baseline and the prevalence of metabolic syndrome at follow-up (mean follow-up period, 3.9 years). Similarly, logistic regression analyses for risk of abdominal obesity, obesity, hypertension, dyslipidemia (hypertriglyceridemia), low HDL, and hyperglycemia were performed after excluding individuals with the respective condition at baseline.

Multivariable adjustments were conducted for sex, age, BMI, smoking, alcohol consumption, physical activity, breakfast intake, dyslipidemia, hypertension, and hyperglycemia ("present" or "none" for all items). In the case of one, or both, of the above physical activities and exercises, we referred to them broadly as physical activity in logistic regression analysis. After adjusting for these risk factors, we tested effect modification by sex using an interaction term generated by multiplying variables of age, BMI, smoking, alcohol consumption, physical activity, breakfast intake, dyslipidemia, hypertension, and hyperglycemia. Moreover, we determined whether there was an additive interaction of both night eating habits on the development of metabolic syndrome and 
its components. We calculated the relative excess risk due to interaction (RERI), which is the excess risk as a result of joint exposure, as the odds ratio ("dinner immediately before bed" + "snacks after dinner") - the odds ratio ("dinner immediately before bed") - the odds ratio ("snacks after dinner") +1 [6]. We further tested the hypothesis of the RERI $=0$ using the Z-test and converted the values to $p$-values [23]. RERI scores $>0$ suggest a synergism or greater risk due to interaction than the additive effects of each of both factors [24, 25]. Moreover, the percentage RERI (RERI\%), defined as the proportion of disease burden caused by the interaction of two factors [6], was calculated using the following formula: (RERI/odds ratio ["dinner immediately before bed" + "snacks after dinner"]) $\times 100$.

A significance level of 0.05 (two-tailed) was used for all statistical analyses. The SAS statistical software ver. 9.4 (SAS Institute Inc., Cary, NC, USA) was used to conduct all statistical analyses.

\section{Results}

\section{Participant characteristics}

Table 1 shows the mean age, age-adjusted mean values (standard errors), and proportion of baseline characteristics according to the presence of both night eating habits. Compared to participants with neither habit, participants with both habits had a higher BMI, waist circumference, and LDL cholesterol level $(p<0.0001$ for all). Moreover, a higher proportion of participants with both habits reported skipping breakfast $\geq 3$ times/week.

\section{Metabolic syndrome, lifestyle-related behaviors, and biochemical analysis results}

Table 2 describes the rates of metabolic syndrome and lifestyle-related behaviors as well as the mean values (standard deviations) of the biochemical analysis results at baseline and follow-up. In participants, the prevalence of metabolic syndrome was $2.5 \%$ higher at follow-up than at baseline $(p<0.0001)$. Mean BMI, waist circumference, systolic blood pressure, diastolic blood pressure, and $\mathrm{HbA1c}$ levels were also higher at follow-up than at baseline in participants $(p<0.0001$ for all). Mean triglyceride and LDL cholesterol levels increased in women and decreased in men ( $\mathrm{p}<0.0001$ for all). Regarding lifestyle-related behaviors, the rates of current smoking $(\mathrm{p}<0.0001)$ and skipping breakfast $(p=0.002)$ decreased in participants, whereas the rates of exercise and physical activity increased ( $p<0.0001$ for both) over time. The rates of current drinking and excessive drinking increased in men ( $\mathrm{p}<0.0001$ for both) and only the rate of excessive drinking increased in women $(\mathrm{p}<0.0001)$. Although improvements over time were observed for most lifestyle-related behaviors (except for drinking) in participants, no changes were seen for "dinner immediately before bed" (24.6\% and $23.9 \%$ at baseline and follow-up, respectively; $p=0.203)$ and "snacks after dinner" (11.9\% and $11.8 \%$ at baseline and follow-up, respectively; $p=$ $0.559)$.

\section{Relationship between night eating habits and metabolic syndrome}

Tables 3 and 4 show the relationships between night eating habits at baseline and metabolic syndrome (and its components) at follow-up. At follow-up, 478 individuals (289 men and 189 women) had developed metabolic syndrome. Women with both night eating habits had higher odds of developing metabolic syndrome than those with neither habit (multivariable-adjusted OR, 1.68; $95 \%$ CI, $1.00-2.84$ ). No supra-additive interaction of both habits on the development of metabolic syndrome was observed; moreover, the association between both night eating habits and metabolic syndrome did not vary significantly by sex. In men, we did not find a significant association between night eating habits and metabolic syndrome.

\section{Relationship between night eating habits and abdominal obesity}

Men and women with both night eating habits had higher odds of abdominal obesity than those with neither habit (multivariable-adjusted OR, 1.42; 95\% CI, 1.03-1.97). However, the association between both night eating habits and abdominal obesity did not vary significantly by sex.

\section{Relationship between night eating habits and obesity (body mass index $\geq 25 \mathrm{~kg} / \mathrm{m}^{2}$ )}

When obesity was evaluated using BMI $\geq 25 \mathrm{~kg} / \mathrm{m}^{2}$ as the cut-off, we observed that participants with both night eating habits had higher odds of developing obesity than those with neither habit (multivariable-adjusted OR, 2.11 ; 95\% CI, $1.42-3.15$ for men and 3.02; 95\% CI, $1.72-5.29$ for women). The presence of both night eating habits appeared to have a supra-additive interaction of borderline significance for obesity in women (RERI, 1.67; RERI\%, 85.0\%; $p=0.058$ ). In men, each habit ("dinner immediately before bed" and "snacks after dinner") was associated with increased odds for developing obesity.

\section{Relationship between night eating habits and hypertension}

Participants with both night eating habits had lower odds of developing hypertension than those with neither habit (multivariable-adjusted OR, 0.78; 95\% CI, 0.591.03). However, this association was not significant. 
Table 1 Age-adjusted mean values and baseline characteristics according to night eating habits at baseline (2009-2010). Study undertaken in Okayama

\begin{tabular}{|c|c|c|c|c|c|}
\hline & Total & $\begin{array}{l}\text { Those with } \\
\text { neither habit }\end{array}$ & $\begin{array}{l}\text { Those with a single } \\
\text { habit of "dinner } \\
\text { immediately before } \\
\text { bed" ( } \geq 3 \text { times per week) }\end{array}$ & $\begin{array}{l}\text { Those with a single } \\
\text { habit of "snacks after } \\
\text { dinner" ( } \geq 3 \text { times } \\
\text { per week) }\end{array}$ & $\begin{array}{l}\text { Those with } \\
\text { both habits }\end{array}$ \\
\hline \multicolumn{6}{|l|}{ Total } \\
\hline Number of participants ( $\mathrm{n}$ ) & 8153 & 4462 & 2006 & 972 & 713 \\
\hline Mean (SD) Age (years) & 46.8 & $47.0(0.1)$ & $46.3(0.1)^{*}$ & $47.1(0.2)$ & $46.5(0.2)^{*}$ \\
\hline $\mathrm{BMI}\left(\mathrm{kg} / \mathrm{m}^{2}\right)$ & 22.9 & $22.7(0.0)$ & $22.9(0.1)$ & $23.1(0.1)^{*}$ & $23.9(0.1)^{*}$ \\
\hline Waist circumference $(\mathrm{cm})$ & 81.7 & $81.2(0.1)$ & $81.6(0.2)$ & $81.9(0.3)$ & $84.2(0.3)^{*}$ \\
\hline Systolic blood pressure (mmHg) & 117.6 & $117.8(0.2)$ & $117.5(0.3)$ & $116.8(0.5)$ & $117.2(0.6)$ \\
\hline Diastolic blood pressure $(\mathrm{mmHg})$ & 75.3 & $75.3(0.2)$ & $75.6(0.3)$ & $74.8(0.4)$ & $75.0(0.4)$ \\
\hline Triglycerides (mg/dl) & 111.6 & $111.5(1.2)$ & $111.2(1.8)$ & $109.6(2.6)$ & $115.6(3.0)$ \\
\hline HDL cholesterol (mg/dl) & 64.8 & $64.7(0.2)$ & $65.5(0.3)$ & $64.4(0.5)$ & $63.9(0.6)$ \\
\hline LDL cholesterol (mg/dl) & 128.1 & $127.0(0.5)$ & $127.9(0.7)$ & $129.8(1.0)^{*}$ & $133.6(1.2)^{*}$ \\
\hline HbA1c (NGSP values) (\%) & 5.54 & $5.52(0.01)$ & $5.56(0.01)$ & $5.57(0.02)$ & $5.56(0.02)$ \\
\hline Metabolic syndrome n (\%) & $866(10.6)$ & $399(8.9)$ & $248(12.4 \%)^{*}$ & $113(11.6)^{*}$ & $106(14.9)^{*}$ \\
\hline Current smokers $n(\%)^{a}$ & $2134(28.1)$ & $1022(24.7)$ & $732(38.8 \%)^{*}$ & $173(19.3)$ & $207(31.0)$ \\
\hline $\begin{array}{l}\text { "I have been engaged in light } \geq 30 \text {-min exercise } \\
\text { more than twice a week for }>1 \text { year" (\%) }{ }^{\text {a }}\end{array}$ & $1603(21.1)$ & $908(22.0)$ & $381(20.2 \%)^{*}$ & $186(20.7)$ & $128(19.2)$ \\
\hline "Physical activity for $>1$ h/day" $n(\%)^{a}$ & $1258(16.6)$ & $693(16.8)$ & $311(16.5 \%)$ & $151(16.8)$ & $103(15.4)$ \\
\hline "I skip breakfast > 3 times a week." n (\%) ${ }^{\text {a }}$ & $1219(16.1)$ & $486(11.8)$ & $448(23.7 \%)^{*}$ & $107(11.9)$ & $178(26.7)^{*}$ \\
\hline Current drinkers n (\%) & $4582(56.2)$ & $2360(52.9)$ & $1429(71.2 \%)^{*}$ & $398(41.0)^{*}$ & $395(55.4)$ \\
\hline Excessive drinkers n (\%) & $968(11.9)$ & $438(9.8)$ & $364(18.2 \%)^{*}$ & $64(6.6)$ & $102(14.3)$ \\
\hline \multicolumn{6}{|l|}{ Male } \\
\hline Number of participants ( $n$ ) & 4875 & 2357 & 1619 & 403 & 496 \\
\hline Mean (SD) Age (years) & 46.9 & $47.2(0.1)$ & $46.5(0.1)^{*}$ & $47.0(0.2)$ & $46.5(0.2)^{*}$ \\
\hline $\mathrm{BMI}\left(\mathrm{kg} / \mathrm{m}^{2}\right)$ & 23.7 & $23.6(0.1)$ & $23.5(0.1)$ & $24.0(0.2)$ & $24.6(0.1)^{*}$ \\
\hline Waist circumference $(\mathrm{cm})$ & 84.5 & $84.1(0.2)$ & $84.3(0.2)$ & $85.2(0.4)$ & $86.9(0.4)^{*}$ \\
\hline Systolic blood pressure (mmHg) & 120.5 & $121.0(0.3)$ & $120.4(0.4)$ & 119.4(0.7) & 119.7(0.7) \\
\hline Diastolic blood pressure $(\mathrm{mmHg})$ & 77.2 & $77.4(0.2)$ & $77.4(0.3)$ & $76.0(0.6)$ & $76.5(0.5)$ \\
\hline Triglycerides (mg/dl) & 133.0 & $133.6(2.0)$ & $131.6(2.4)$ & $129.8(4.9)$ & $137.4(4.4)$ \\
\hline HDL cholesterol (mg/dl) & 59.4 & $59.3(0.3)$ & $60.3(0.4)$ & $57.8(0.7)$ & $58.4(0.7)$ \\
\hline LDL cholesterol (mg/dl) & 131.6 & $130.2(0.7)$ & $131.3(0.8)$ & $135.6(1.6)^{*}$ & $136.3(1.4)^{*}$ \\
\hline HbA1c (NGSP values) (\%) & 5.58 & $5.55(0.01)$ & $5.59(0.02)$ & $5.63(0.03)$ & $5.60(0.03)$ \\
\hline Metabolic syndrome n (\%) & $613(12.6)$ & $259(11.0)$ & $207(12.8)$ & $63(15.6)^{*}$ & $84(16.9)^{*}$ \\
\hline Current smokers $n(\%)^{b}$ & $1869(41.2)$ & $869(39.7)$ & $684(45.0)^{*}$ & $138(37.5)$ & $178(38.7)$ \\
\hline $\begin{array}{l}\text { "I have been engaged in light } \geq 30 \text {-min exercise } \\
\text { more than twice a week for }>1 \text { year" } n(\%)^{b}\end{array}$ & $1097(24.2)$ & $585(26.7)$ & $331(21.8)^{*}$ & $83(22.6)$ & $98(21.3)$ \\
\hline "Physical activity for $>1$ h/day" n (\%) & $820(18.1)$ & $411(18.8)$ & $268(17.6)$ & $69(18.8)$ & $72(15.7)$ \\
\hline "I skip breakfast > 3 times a week." n (\%) & $920(20.3)$ & $337(15.4)$ & $378(24.9)^{*}$ & $66(17.9)$ & $139(30.2)^{*}$ \\
\hline Current drinkers n (\%) & $3368(69.1)$ & $1605(68.1)$ & $1226(75.7)^{*}$ & $226(56.1)^{*}$ & $311(62.7)$ \\
\hline Excessive drinkers n (\%) & $878(18.0)$ & $388(16.5)$ & $345(21.3)^{*}$ & $53(13.2)$ & $92(18.6)$ \\
\hline \multicolumn{6}{|l|}{ Female } \\
\hline Number of participants (n) & 3278 & 2105 & 387 & 569 & 217 \\
\hline Mean (SD) Age (years) & 46.7 & $46.7(0.1)$ & $45.9(0.2)^{*}$ & $47.0(0.2)$ & $46.5(0.3)$ \\
\hline $\mathrm{BMI}\left(\mathrm{kg} / \mathrm{m}^{2}\right)$ & 21.7 & $21.5(0.1)$ & $22.1(0.2)^{*}$ & $21.8(0.1)$ & $22.9(0.2)^{*}$ \\
\hline
\end{tabular}


Table 1 Age-adjusted mean values and baseline characteristics according to night eating habits at baseline (2009-2010). Study undertaken in Okayama (Continued)

\begin{tabular}{|c|c|c|c|c|c|}
\hline & Total & $\begin{array}{l}\text { Those with } \\
\text { neither habit }\end{array}$ & $\begin{array}{l}\text { Those with a single } \\
\text { habit of "dinner } \\
\text { immediately before } \\
\text { bed" ( } \geq 3 \text { times per week) }\end{array}$ & $\begin{array}{l}\text { Those with a single } \\
\text { habit of "snacks after } \\
\text { dinner" ( } \geq 3 \text { times } \\
\text { per week) }\end{array}$ & $\begin{array}{l}\text { Those with } \\
\text { both habits }\end{array}$ \\
\hline Waist circumference $(\mathrm{cm})$ & 77.4 & $76.9(0.2)$ & $78.0(0.5)$ & $77.4(0.4)$ & $80.4(0.6)^{*}$ \\
\hline Systolic blood pressure $(\mathrm{mmHg})$ & 113.1 & $113.1(0.3)$ & $113.4(0.8)$ & $112.7(0.6)$ & $113.7(1.0)$ \\
\hline Diastolic blood pressure $(\mathrm{mmHg})$ & 72.5 & $72.4(0.2)$ & $72.8(0.5)$ & $72.5(0.4)$ & $72.9(0.7)$ \\
\hline Triglycerides (mg/dl) & 79.7 & $79.0(0.9)$ & $83.1(2.1)$ & $78.6(1.7)$ & $82.7(2.8)$ \\
\hline HDL cholesterol (mg/dl) & 72.8 & $72.8(0.3)$ & $72.5(0.8)$ & $73.3(0.7)$ & $72.3(1.1)$ \\
\hline LDL cholesterol (mg/dl) & 122.8 & $122.2(0.6)$ & $122.6(1.5)$ & $122.8(1.2)$ & $129.8(2.0)^{*}$ \\
\hline HbA1c (NGSP values) (\%) & 5.48 & $5.47(0.01)$ & $5.51(0.02)$ & $5.49(0.02)$ & $5.49(0.03)$ \\
\hline Metabolic syndrome $n(\%)$ & $253(7.7)$ & $140(6.7)$ & $41(10.6)^{*}$ & $50(8.8)$ & $22(10.1)$ \\
\hline Current smokers $\mathrm{n}(\%)^{\mathrm{c}}$ & $265(8.7)$ & $153(7.9)$ & $48(13.1)^{*}$ & $35(6.6)$ & $29(13.9)^{*}$ \\
\hline $\begin{array}{l}\text { "I have been engaged in light } \geq 30 \text {-min exercise } \\
\text { more than twice a week for }>1 \text { year" } n(\%)^{c}\end{array}$ & $506(16.6)$ & $323(16.6)$ & $50(13.6)$ & $103(19.5)$ & $30(14.4)$ \\
\hline "Physical activity for $>1$ h/day" n (\%) & $438(14.4)$ & $282(14.5)$ & $43(11.7)$ & $82(15.5)$ & $31(14.9)$ \\
\hline "I skip breakfast $>3$ times a week." n (\%) & $299(9.8 \%)$ & $149(7.7)$ & $70(19.1)^{*}$ & $41(7.8)$ & $39(18.8)^{*}$ \\
\hline Current drinkers n (\%) & $1214(37.0)$ & $755(35.9)$ & $203(52.5)^{*}$ & $172(30.2)^{*}$ & $84(38.7)$ \\
\hline Excessive drinkers n (\%) & $90(2.8)$ & $50(2.4)$ & $19(4.9)^{*}$ & $11(1.9)$ & $10(4.6)$ \\
\hline
\end{tabular}

" $p<0.05$ Comparison of the group with a single habit of either "dinner immediately before bed" or "snacks after dinner", or the group with both habits, to the group without both habits. $B M I$, body mass index; $H D L$, high-density lipoprotein; $L D L$, low-density lipoprotein; HbA1c, glycated hemoglobin; NGSP, National Glycohemglobin Standardization Program

\section{Relationship between night eating habits and dyslipidemia}

Men with both night eating habits had a greater probability of developing dyslipidemia (hypertriglyceridemia) than those with neither habit (multivariable-adjusted OR, 1.46; 95\% CI, 1.06-2.01 and 1.61; 95\% CI, 1.142.27 , respectively). Women with both night eating habits had higher odds of dyslipidemia than those with neither habit (multivariable-adjusted OR, 1.66; 95\% CI, 1.062.61). The association between both night eating habits and dyslipidemia did not vary significantly by sex.

\section{Relationship between night eating habits and hyperglycemia}

Women reporting only "dinner immediately before bed $\geq 3$ times/week" had lower odds of hyperglycemia than those reporting neither night eating habit.

\section{Discussion}

Participants reporting both night eating habits ("dinner immediately before bed" and "snacks after dinner") had higher odds for obesity at follow-up than at baseline. Moreover, we detected a supra-additive interaction of borderline significance for obesity in women with both habits. In men and women, having both night eating habits was associated with dyslipidemia. In men, it was associated with hypertriglyceridemia. Furthermore, sex differences did not seem to affect the prevalence of metabolic syndrome and its components, as the tests for effect modification by sex were not significant in those with both night eating habits.

A previous randomized crossover trial that compared the effects of night and day snack intake of approximately $200 \mathrm{kcal}$ daily for 13 days showed that the blood LDL cholesterol level was higher in the night time snack intake group than in the daytime snack intake group [26]. An epidemiological study of 150 male bus drivers indicated that night workers had a higher risk of obesity $\left(B M I \geq 25 \mathrm{~kg} / \mathrm{m}^{2}\right.$ ) and abdominal obesity (waist circumference $\geq 94 \mathrm{~cm}$ ) than daytime workers [27]. Furthermore, having dinner immediately before bed was reported to be significantly associated with metabolic syndrome and hyperglycemia $[13,28]$, and men who ate snacks after dinner had a higher risk of coronary heart disease than men who did not [29]. A previous study showed an association between "dinner immediately before bed" and metabolic syndrome in women, but not men [13].

In our study, men and women with both night eating habits showed higher ORs for obesity than those without either habit. Noticeably, both habits tended to show a supra-additive interaction for developing obesity in women, although this result was not statistically significant (RERI, 1.67; RERI\%, 85.0\%; $p=0.058$ ). In men, each habit ("dinner immediately before bed" and "snacks after dinner") was associated with higher odds of developing obesity. Moreover, men with both night eating habits 
Table 2 Rate of metabolic syndrome at baseline (2009-2010) and after an average of 3.9 years. Study undertaken in Okayama

\begin{tabular}{|c|c|c|c|c|c|c|c|c|c|}
\hline & \multicolumn{2}{|l|}{ Total } & \multirow[t]{2}{*}{$p$ value } & \multicolumn{2}{|l|}{ Male } & \multirow[t]{2}{*}{$p$ value } & \multicolumn{2}{|l|}{ Female } & \multirow[t]{2}{*}{$p$ value } \\
\hline & 2009-2010 & 2013-2014 & & 2009-2010 & $2013-2014$ & & $2009-2010$ & $2013-2014$ & \\
\hline Number of participants $(N)$ & 8153 & 8153 & - & 4875 & 4875 & - & 3278 & 3278 & - \\
\hline Age (years) & $46.8(4.6)$ & $50.6(4.9)$ & $<0.0001$ & $46.9(4.8)$ & $50.7(4.9)$ & $<0.0001$ & $46.7(4.7)$ & $50.5(4.8)$ & $<0.0001$ \\
\hline $\mathrm{BMI}\left(\mathrm{kg} / \mathrm{m}^{2}\right)$ & $22.9(3.4)$ & $23.1(3.5)$ & $<0.0001$ & $23.7(3.3)$ & $23.8(3.3)$ & $<0.0001$ & $21.7(3.3)$ & $22.0(3.5)$ & $<0.0001$ \\
\hline Waist circumference $(\mathrm{cm})$ & $81.7(9.6)$ & $82.3(9.6)$ & $<0.0001$ & $84.5(8.9)$ & $84.9(9.0)$ & $<0.0001$ & $77.4(9.0)$ & $78.5(9.3)$ & $<0.0001$ \\
\hline Systolic blood pressure $(\mathrm{mmHg})$ & $117.6(15.4)$ & $118.8(16.6)$ & $<0.0001$ & $120.5(15.0)$ & $121.8(15.7)$ & $<0.0001$ & $113.1(15.0)$ & $114.2(16.7)$ & $<0.0001$ \\
\hline Diastolic blood pressure $(\mathrm{mmHg})$ & $75.3(11.4)$ & $75.9(11.1)$ & $<0.0001$ & $77.2(11.3)$ & $78.1(10.7)$ & $<0.0001$ & $72.5(10.9)$ & $72.8(11.1)$ & 0.044 \\
\hline Triglycerides (mg/dl) & $111.6(84.2)$ & $109.8(84.6)$ & 0.004 & $133.0(97.6)$ & $127.5(98.6)$ & $<0.0001$ & $79.7(42.0)$ & $83.4(46.8)$ & $<0.0001$ \\
\hline HDL cholesterol (mg/dl) & $64.8(16.3)$ & $65.0(17.4)$ & 0.356 & $59.4(14.5)$ & $59.4(15.5)$ & 0.145 & $72.8(15.6)$ & $73.5(16.6)$ & 0.002 \\
\hline LDL cholesterol (mg/dl) & $128.1(31.9)$ & $127.7(31.3)$ & $<0.0001$ & $131.6(32.0)$ & $128.5(31.1)$ & $<0.0001$ & $122.8(30.9)$ & $126.6(31.6)$ & $<0.0001$ \\
\hline HbA1c (NGSP values) $n(\%)$ & $5.54(0.6)$ & $5.59(0.6)$ & $<0.0001$ & $5.58(0.7)$ & $5.63(0.6)$ & $<0.0001$ & $5.48(0.4)$ & $5.53(0.5)$ & $<0.0001$ \\
\hline Metabolic syndrome $n(\%)$ & $866(10.6 \%)$ & $1066(13.1)$ & $<0.0001$ & $613(12.6)$ & $702(14.4)$ & $<0.0001$ & $253(7.7)$ & $364(11.1)$ & $<0.0001$ \\
\hline Current smokers $n(\%)^{a}$ & $2134(28.1)$ & $1760(23.2)$ & $<0.0001$ & $1869(41.2)$ & $1539(33.9)$ & $<0.0001$ & $265(8.7)$ & $221(7.3)$ & $<0.0001$ \\
\hline $\begin{array}{l}\text { "I have been engaged in light } \\
\geq 30 \text {-min exercise more than } \\
\text { twice a week for }>1 \\
\text { year" } n(\%)^{\text {a }}\end{array}$ & $1603(21.1)$ & $1847(24.4)$ & $<0.0001$ & $1097(24.2)$ & $1249(27.5)$ & $<0.0001$ & $506(16.6)$ & 598 (19.6) & $<0.0001$ \\
\hline $\begin{array}{l}\text { "Physical activity for > } 1 \text { h/ } \\
\text { day" } n(\%)^{\mathrm{a}}\end{array}$ & $1258(16.6)$ & 1409 (18.6) & $<0.0001$ & $820(18.1)$ & $920(20.3)$ & 0.0007 & $438(14.4)$ & $489(16.1)$ & 0.020 \\
\hline $\begin{array}{l}\text { Those with a single habit of } \\
\text { "dinner within } 2 \mathrm{~h} \text { of } \\
\text { bedtime" ( } \geq 3 \text { times } \\
\text { per week) } n(\%)\end{array}$ & 2006 (24.6) & $1952(23.9)$ & 0.203 & $1619(33.2)$ & $1543(31.7)$ & 0.036 & $387(11.8)$ & 409 (12.5) & 0.320 \\
\hline $\begin{array}{l}\text { Those with a single habit of } \\
\text { "snacks after dinner" } \\
(\geq 3 \text { times per week) } \\
n(\%)\end{array}$ & $972(11.9)$ & $962(11.8)$ & 0.559 & $403(8.3)$ & $401(8.2)$ & 0.488 & $569(17.4)$ & $561(17.1)$ & 0.888 \\
\hline $\begin{array}{l}\text { Those with both habits of } \\
\text { "dinner within } 2 \mathrm{~h} \text { of } \\
\text { bedtime" and "snacks } \\
\text { after dinner" ( } \geq 3 \text { times } \\
\text { per week) } n(\%)\end{array}$ & $713(8.8)$ & $694(8.5)$ & 0.168 & $496(10.2)$ & $489(10.0)$ & 0.468 & $217(6.6)$ & $205(6.3)$ & 0.213 \\
\hline $\begin{array}{l}\text { "I skip breakfast > } 3 \text { times } \\
\text { a week." } n(\%)^{\mathrm{a}}\end{array}$ & $1219(16.1)$ & $1134(15.0)$ & 0.002 & $920(20.3)$ & $836(18.4)$ & 0.0001 & $299(9.8)$ & $298(9.8)$ & 1.000 \\
\hline Current drinkers $n(\%)$ & $4582(56.2)$ & $4683(57.4)$ & 0.002 & $3368(69.1)$ & $3475(71.3)$ & $<0.0001$ & 1214(37.0) & $1208(36.9)$ & 0.817 \\
\hline excessive drinkers $n(\%)$ & $968(11.9)$ & $1238(15.2)$ & $<0.0001$ & $878(18.0)$ & $1109(22.8)$ & $<0.0001$ & $90(2.8)$ & 129 (3.9) & 0.0006 \\
\hline
\end{tabular}

BMI, waste circumference, blood pressure, and biochemical analysis results were compared using the Wilcoxon signed rank-tests. The prevalence of metabolic syndrome, current smoker, physical activity, eating habits, current drinker and excessive drinker were compared using McNemar's test. $B M I$, body mass index; $H D L$, high-density lipoprotein; $L D L$, low-density lipoprotein; HbA1c; glycated hemoglobin; NGSP, National Glycohemoglobin Standardization Program

had higher odds for dyslipidemia (hypertriglyceridemia) when compared to those with neither habit.

There are several explanations for the observed association between night eating habits and increased odds for developing metabolic syndrome and its component in women. Late-night eating could lead to circadian misalignment, reduced energy expenditure (reduced levels of leptin), increased appetite sensations, and weight gain [30]. In addition to behavioral chronodisruption, circadian gene variations have been associated with altered metabolism resulting in negative health outcomes. For example, clock gene mutation carriers, in the minor $\mathrm{C}$ allele, were resistant to weight loss after a 12-14-week intervention. In addition, they had shorter sleep durations, higher ghrelin concentrations, and reported a preference for delayed breakfast time and evening meals [31]. Energy expenditure is lower at night than during the day $[15,32]$. Therefore, when compared to daytime snacking, night time snacking has been associated with decreased fat oxidation [33] and can cause fat accumulation because nutrients ingested at night are not used for glycogen synthesis in muscles and the liver [34]. Shimba et al. reported that the circadian clock gene BMAL1, which regulates circadian rhythms and adipogenesis, is primarily activated from $10 \mathrm{PM}$ to $2 \mathrm{AM}$, and that the overexpression of BMAL1 in adipocytes increases lipid 
Table 3 Relationship between two night eating habits and metabolic syndrome after 3.9 years (2013-2014). Study undertaken in Okayama

\begin{tabular}{|c|c|c|c|c|}
\hline & Total & & & \\
\hline & $\begin{array}{l}\text { Those with } \\
\text { neither habit }\end{array}$ & $\begin{array}{l}\text { Those with a single habit of } \\
\text { "dinner immediately before bed" } \\
\text { ( } \geq 3 \text { times per week) }\end{array}$ & $\begin{array}{l}\text { Those with a single habit } \\
\text { of "snacks after dinner" } \\
\text { ( } \geq 3 \text { times per week) }\end{array}$ & $\begin{array}{l}\text { Those with } \\
\text { both habits }\end{array}$ \\
\hline Metabolic syndrome & $n=7287$ & & & \\
\hline Number of participants & $249 / 4063$ & $120 / 1758$ & $58 / 859$ & $51 / 607$ \\
\hline Model $1^{a}$ & 1.00 & $1.14(0.90-1.44)$ & $1.12(0.83-1.51)$ & $1.43(1.04-1.96)$ \\
\hline Model $2^{b}$ & 1.00 & $1.10(0.86-1.41)$ & $1.13(0.83-1.54)$ & $1.33(0.95-1.86)$ \\
\hline Abdominal obesity & $n=5780$ & & & \\
\hline Number of participants & $342 / 3212$ & $148 / 1485$ & $87 / 652$ & $58 / 431$ \\
\hline Model 1 & 1.00 & $1.20(0.97-1.49)$ & $1.18(0.91-1.52)$ & $1.52(1.12-2.07)$ \\
\hline Model 2 & 1.00 & $1.15(0.92-1.45)$ & $1.19(0.91-1.55)$ & $1.41(1.03-1.95)$ \\
\hline Model $3^{c}$ & 1.00 & $1.16(0.93-1.47)$ & $1.19(0.91-1.55)$ & $1.42(1.03-1.97)$ \\
\hline Obesity & $n=6225$ & & & \\
\hline Number of participants & $204 / 3542$ & $128 / 1489$ & $51 / 747$ & $63 / 447$ \\
\hline Model 1 & 1.00 & $1.32(1.04-1.68)$ & $1.30(0.94-1.79)$ & $2.47(1.82-3.35)$ \\
\hline Model 2 & 1.00 & $1.31(1.01-1.69)$ & $1.32(0.95-1.84)$ & $2.34(1.69-3.23)$ \\
\hline Model $3^{c}$ & 1.00 & $1.33(1.02-1.71)$ & $1.32(0.95-1.84)$ & $2.37(1.71-3.29)$ \\
\hline Hypertension & $n=6074$ & & & \\
\hline Number of participants & $512 / 3368$ & $252 / 1431$ & $103 / 749$ & $80 / 526$ \\
\hline Model 1 & 1.00 & $1.04(0.87-1.23)$ & $0.95(0.75-1.19)$ & $0.92(0.71-1.19)$ \\
\hline Model 2 & 1.00 & $0.97(0.81-1.17)$ & $0.95(0.74-1.21)$ & $0.89(0.68-1.16)$ \\
\hline Model $3^{d}$ & 1.00 & $0.98(0.82-1.18)$ & $0.92(0.72-1.17)$ & $0.78(0.59-1.03)$ \\
\hline Dyslipidemia & $n=6177$ & & & \\
\hline Number of participants & $447 / 3462$ & $239 / 1450$ & $96 / 763$ & $102 / 502$ \\
\hline Model 1 & 1.00 & $1.16(0.97-1.39)$ & $1.04(0.82-1.31)$ & $1.63(1.28-2.08)$ \\
\hline Model 2 & 1.00 & $1.20(0.99-1.45)$ & $1.07(0.83-1.37)$ & $1.61(1.25-2.09)$ \\
\hline Model $3^{\mathrm{e}}$ & 1.00 & $1.18(0.97-1.43)$ & $1.03(0.80-1.33)$ & $1.49(1.14-1.94)$ \\
\hline Hyper triglyceridemia & $n=6605$ & & & \\
\hline Number of participants & $286 / 3694$ & $176 / 1535$ & $64 / 833$ & $71 / 543$ \\
\hline Model 1 & 1.00 & $1.21(0.98-1.49)$ & $1.11(0.83-1.47)$ & $1.58(1.19-2.09)$ \\
\hline Model 2 & 1.00 & $1.15(0.92-1.42)$ & $1.16(0.87-1.56)$ & $1.44(1.07-1.95)$ \\
\hline Model $3^{\mathrm{e}}$ & 1.00 & $1.13(0.91-1.41)$ & $1.11(0.83-1.49)$ & $1.35(0.99-1.84)$ \\
\hline Low HDL cholesterolemia & $n=7750$ & & & \\
\hline Number of participants & $125 / 4246$ & $62 / 1922$ & $27 / 917$ & $32 / 665$ \\
\hline Model 1 & 1.00 & $1.13(0.82-1.56)$ & $0.99(0.65-1.52)$ & $1.70(1.14-2.53)$ \\
\hline Model 2 & 1.00 & $1.27(0.91-1.79)$ & $1.07(0.69-1.67)$ & $1.63(1.06-2.51)$ \\
\hline Model $3^{\mathrm{e}}$ & 1.00 & $1.19(0.84-1.68)$ & $0.99(0.64-1.55)$ & $1.36(0.88-2.11)$ \\
\hline Hyperglycemia & $n=5717$ & & & \\
\hline Number of participants & $509 / 3194$ & 198/1386 & $106 / 669$ & $79 / 468$ \\
\hline Model 1 & 1.00 & $0.96(0.80-1.15)$ & $0.97(0.77-1.22)$ & $1.16(0.89-1.51)$ \\
\hline Model 2 & 1.00 & $0.98(0.81-1.19)$ & $0.92(0.72-1.18)$ & $1.13(0.86-1.49)$ \\
\hline Model $3^{f}$ & 1.00 & $0.99(0.82-1.20)$ & $0.90(0.70-1.15)$ & $1.06(0.80-1.40)$ \\
\hline
\end{tabular}

Overall analysis was adjusted by sex.

${ }^{a}$ Adjusted by age

${ }^{\mathrm{b}}$ Adjusted by age, smoking habit (yes, no, missing), alcohol consumption (yes, no), physical activity (yes, no, missing), and breakfast intake (yes, no, missing)

'Adjusted by age, smoking habit, alcohol consumption, physical activity, breakfast intake, hypertension, dyslipidemia, and hyperglycemia

${ }^{\mathrm{d}}$ Adjusted by age, smoking habit, alcohol consumption, physical activity, breakfast intake, dyslipidemia, hyperglycemia, and BMI

${ }^{\mathrm{e} A d j u s t e d ~ b y ~ a g e, ~ s m o k i n g ~ h a b i t, ~ a l c o h o l ~ c o n s u m p t i o n, ~ p h y s i c a l ~ a c t i v i t y, ~ b r e a k f a s t ~ i n t a k e, ~ h y p e r t e n s i o n, ~ h y p e r g l y c e m i a, ~ a n d ~ B M I ~}$

${ }^{f}$ Adjusted by age, smoking habit, alcohol consumption, physical activity, breakfast intake, hypertension, dyslipidemia, and BMI

$H D L$, high-density lipoprotein 
Table 4 Relationship between two night eating habits and metabolic syndrome after 3.9 years (2013-2014) by sex. Study undertaken in Okayama

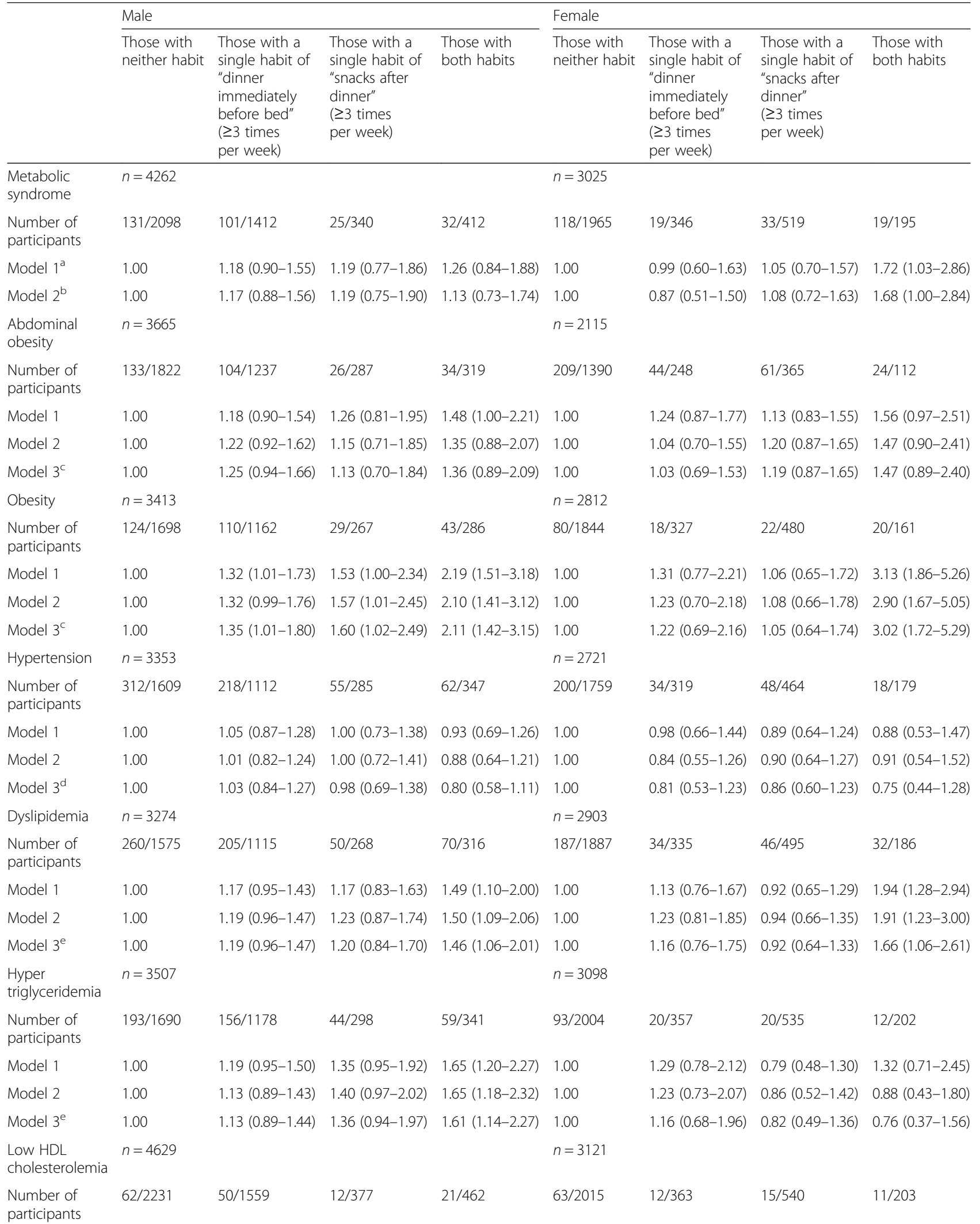


Table 4 Relationship between two night eating habits and metabolic syndrome after 3.9 years (2013-2014) by sex. Study undertaken in Okayama (Continued)

\begin{tabular}{|c|c|c|c|c|c|c|c|c|}
\hline & \multicolumn{4}{|l|}{ Male } & \multicolumn{4}{|l|}{ Female } \\
\hline & $\begin{array}{l}\text { Those with } \\
\text { neither habit }\end{array}$ & $\begin{array}{l}\text { Those with a } \\
\text { single habit of } \\
\text { "dinner } \\
\text { immediately } \\
\text { before bed" } \\
\text { ( } \geq 3 \text { times } \\
\text { per week) }\end{array}$ & $\begin{array}{l}\text { Those with a } \\
\text { single habit of } \\
\text { "snacks after } \\
\text { dinner" } \\
\text { ( } \geq 3 \text { times } \\
\text { per week) }\end{array}$ & $\begin{array}{l}\text { Those with } \\
\text { both habits }\end{array}$ & $\begin{array}{l}\text { Those with } \\
\text { neither habit }\end{array}$ & $\begin{array}{l}\text { Those with a } \\
\text { single habit of } \\
\text { "dinner } \\
\text { immediately } \\
\text { before bed" } \\
\text { ( } \geq 3 \text { times } \\
\text { per week) }\end{array}$ & $\begin{array}{l}\text { Those with a } \\
\text { single habit of } \\
\text { "snacks after } \\
\text { dinner" } \\
\text { ( } \geq 3 \text { times } \\
\text { per week) }\end{array}$ & $\begin{array}{l}\text { Those with } \\
\text { both habits }\end{array}$ \\
\hline Model 1 & 1.00 & $1.14(0.78-1.67)$ & $1.15(0.61-2.15)$ & $1.65(1.00-2.74)$ & 1.00 & $1.08(0.58-2.03)$ & $0.89(0.50-1.57)$ & $1.77(0.92-3.42)$ \\
\hline Model 2 & 1.00 & $1.25(0.84-1.87)$ & $1.13(0.58-2.19)$ & $1.43(0.82-2.50)$ & 1.00 & $1.32(0.69-2.54)$ & $1.03(0.57-1.84)$ & $2.01(1.02-3.94)$ \\
\hline Model $3^{e}$ & 1.00 & $1.19(0.79-1.78)$ & $1.00(0.51-1.97)$ & $1.23(0.70-2.16)$ & 1.00 & $1.23(0.64-2.40)$ & $0.97(0.54-1.75)$ & $1.67(0.84-3.32)$ \\
\hline Hyperglycemia & $n=3322$ & & & & $n=2395$ & & & \\
\hline $\begin{array}{l}\text { Number of } \\
\text { participants }\end{array}$ & $105 / 1645$ & $69 / 1103$ & $17 / 258$ & $23 / 316$ & $64 / 1549$ & $9 / 283$ & $20 / 411$ & 10/152 \\
\hline Model 1 & 1.00 & $1.12(0.91-1.39)$ & $1.12(0.77-1.61)$ & $1.08(0.77-1.52)$ & 1.00 & $0.58(0.39-0.87)$ & $0.89(0.66-1.19)$ & $1.28(0.85-1.95)$ \\
\hline Model 2 & 1.00 & $1.13(0.91-1.42)$ & $1.01(0.68-1.50)$ & $1.00(0.70-1.44)$ & 1.00 & $0.62(0.41-0.94)$ & $0.87(0.64-1.19)$ & $1.35(0.88-2.06)$ \\
\hline Model $3^{f}$ & 1.00 & $1.16(0.93-1.46)$ & $0.99(0.66-1.47)$ & $0.96(0.66-1.38)$ & 1.00 & $0.61(0.40-0.93)$ & $0.84(0.61-1.15)$ & $1.25(0.81-1.94)$ \\
\hline
\end{tabular}

${ }^{\text {aAdjusted by age }}$

${ }^{\mathrm{b}}$ Adjusted by age, smoking habit, alcohol consumption, physical activity, and breakfast intake

cAdjusted by age, smoking habit, alcohol consumption, physical activity, breakfast intake, hypertension, dyslipidemia, and hyperglycemia

${ }^{\mathrm{d} A d j u s t e d ~ b y ~ a g e, ~ s m o k i n g ~ h a b i t, ~ a l c o h o l ~ c o n s u m p t i o n, ~ p h y s i c a l ~ a c t i v i t y, ~ b r e a k f a s t ~ i n t a k e, ~ d y s l i p i d e m i a, ~ h y p e r g l y c e m i a, ~ a n d ~ B M I ~}$

${ }^{\mathrm{e}}$ Adjusted by age, smoking habit, alcohol consumption, physical activity, breakfast intake, hypertension, hyperglycemia, and BMI

${ }^{f}$ Adjusted by age, smoking habit, alcohol consumption, physical activity, breakfast intake, hypertension, dyslipidemia, and BMI

$H D L$, high-density lipoprotein

synthesis activity [35]. Food intake at night might induce the overexpression of BMAL1 and cause visceral fat accumulation. Additionally, once obesity develops, insulin resistance and metabolic inflammation due to the dysfunction of adipocytokine secretion, which includes increased tumor necrosis factor- $\alpha$ and resistin as well as a decreased adiponectin, are triggered and result in metabolic dysfunction [36]. Finally, postprandial glucose, insulin, and triglyceride levels are significantly elevated when consuming meals at night compared to during the day, resulting in diminished insulin sensitivity [37]. Insulin activates lipoprotein lipase and suppresses the secretion of very low-density lipoprotein (VLDL) cholesterol in the liver. Therefore, lower nocturnal insulin sensitivity could be associated with lower lipoprotein lipase activity and higher circulating plasma triglyceride levels [33]. Therefore, eating at night may lead to metabolic syndrome and its components.

The present study did not observe a significant association between night eating habits and the development of hypertension and hyperglycemia. Nevertheless, "dinner immediately before bed" is an important factor that increases the risk of hypertension and hyperglycemia $[28,38]$.

Moreover, we found that the prevalence of lifestyle-related behaviors (smoking, skipping breakfast, and physical activity) improved at follow-up when compared to the baseline, presumably due to the provision of specific health guidance. However, no decrease in the rates of "dinner immediately before bed", "snacks after dinner", and alcohol consumption was observed. For these reasons, interventions and awareness are important for individuals reporting both night eating habits to help reduce further complications.

\section{Study limitations and strengths}

One limitation of the present study was the low followup rate $(46.5 \%)$. This was due to individuals not undergoing the re-examination at follow-up and/or having missing data on night eating habits in the questionnaire. However, we compared the characteristics of participants and non-participants of the follow-up examination and found no major differences in the prevalence of metabolic syndrome and lifestyle-related behaviors as well as for the results of the biochemical analyses. Thus, the effect of attrition seems to have been weak. The questionnaire included data on eating habits but did not include meal content related questions. Therefore, it is possible that healthy snacks were being consumed at night time. To establish a conclusive relation between night time eating habit and metabolic syndrome, snacking habits of men and women with and without metabolic syndrome should have been monitored. This was not done in the study. Another limitation of this study was that data on sleep duration was not available. Previous studies of healthy participants aged 20-30 years reported that short sleep duration increased energy intake and may contribute to obesity [38-42]. Furthermore, in the present study, some female participants were 
approaching menopause. Reduced energy expenditure owing to decreased estrogen secretion [43] and deleterious changes in adipokines levels in postmenopausal women [44] might be considered as risk factors of metabolic syndrome and its components. Moreover, we did not have information on the exact timings of dinner, bedtime, and the intake of dinners and snacks including alcohol. Future studies should assess factors such as sleep duration, menopause, and food intake carefully.

The main strength of this study is that we analyzed the associations between night eating habits and the prevalence metabolic syndrome and its components using a large-scale dataset derived from middle-aged men and women. Our data will help improve the quality of meal guidance, such as how to adopt a nutritional diet and how to improve eating environments.

\section{Conclusions}

Having both night eating habits, "dinner immediately before bed" and "snacks after dinner", was associated with higher BMI in both Japanese men and women. Men with both habits showed higher odds of dyslipidemia when compared to those with neither habit. Women with both habits had higher odds of metabolic syndrome and abdominal obesity when compared to those with neither habit. In men and women, both night eating habits were associated with dyslipidemia. The presence of both habits tended to show a supra-additive interaction on developing obesity in women. Hence, middle-aged individuals should be advised to refrain from both night eating habits to reduce the risk of developing complications associated with metabolic syndrome.

\section{Abbreviations}

BMl: Body mass index; Cl: Confidence interval; HbA1c: Glycated hemoglobin; HDL: High-density lipoprotein; LDL: Low-density lipoprotein; OR: Odds ratio; RERI: Relative excess risk due to interaction; VLDL: Very low-density lipoprotein

\section{Acknowledgements \\ We would like to express our sincere gratitude to Emeritus Professor Nobuyoshi Shimizu, Director of the Junpukai Health Maintenance Center; Ms. Atsuko Ohashi, a public health nurse of the Junpukai Health Maintenance Center Professor Tetsuya Ohira of the Fukushima Medical University; and other staff members involved in this study. Editorial support was provided by Editage.}

\section{Funding}

This research did not receive any specific grant from funding agencies in the public, commercial, or not-for-profit sectors.

\section{Availability of data and materials}

The datasets used and/or analyzed during the current study are available from the corresponding author on reasonable request.

\section{Authors' contributions}

All the authors have contributed to the planning of the analysis, commented on the manuscript text, and approved the submission and publication. KO obtained the data, conceived and designed the study. JY, EE made substantial contributions to conception and design, analysis and interpretation of data; and has been involved in drafting the manuscript.
$\mathrm{KN}, \mathrm{TI}, \mathrm{KO}$ interpreted and critically revised the manuscript. All authors read and approved the final manuscript

\section{Ethics approval and consent to participate}

This study protocol was approved by the ethics committee of the Okayama University (approval number: 1032) and conducted in accordance with the Declaration of Helsinki. Considering the longitudinal nature of the study and large number of participants, verbal or written consent was not obtained from all participants. The study was advertised on posters in medical examination centers for a certain period of time. Patients were asked to participate either through the website or in person. Their participation in the survey was taken as consent to participate. This protocol was approved by the ethics committee. The data were analyzed anonymously.

\section{Consent for publication}

Not applicable.

\section{Competing interests}

The authors declare that they have no competing interests.

\section{Publisher's Note}

Springer Nature remains neutral with regard to jurisdictional claims in published maps and institutional affiliations.

\section{Author details}

${ }^{1}$ Department of Public Health, Okayama University Graduate School of Medicine, Dentistry and Pharmaceutical Sciences, 2-5-1 Shikatacho, Kita-ku, Okayama 700-8558, Japan. ²Department of Nutrition, Faculty of Food Culture, Kurashiki Sakuyo University, Okayama, Japan.

Received: 20 January 2018 Accepted: 26 November 2018

Published online: 11 December 2018

\section{References}

1. Aune D, Sen A, Norat T, Janszky I, Romundstad P, Tonstad S, et al. Body mass index, abdominal fatness, and heart failure incidence and mortality: a systematic review and dose-response meta-analysis of prospective studies. Circulation. 2016;133:639-49.

2. Chei $\mathrm{CL}$, Iso H, Yamagishi $\mathrm{K}$, Inoue M, Tsugane S. Body mass index and weight change since 20 years of age and risk of coronary heart disease among Japanese: the Japan public health center-based study. Int J Obes. 2008;32:144-51.

3. Doi Y, Ninomiya T, Hata J, Yonemoto K, Arima H, Kubo M, et al. Proposed criteria for metabolic syndrome in Japanese based on prospective evidence the Hisayama study. Stroke. 2009;40:1187-94.

4. Wilson PW, D'Agostino RB, Parise H, Sullivan L, Meigs JB. Metabolic syndrome as a precursor of cardiovascular disease and type 2 diabetes mellitus. Circulation. 2005;112:3066-72.

5. Ministry of Health, Labor and Welfare (2016) National Health and Nutrition Survey Japan, 2015. http://www.mhlw.go.jp/bunya/kenkou/eiyou/dl/h27houkoku.pdf. Accessed 15 Jan 2018.

6. Maruyama K, Sato S, Ohira T, Maeda K, Noda H, Kubota Y, et al. The joint impact on being overweight of self reported behaviours of eating quickly and eating until full: cross sectional survey. BMJ. 2008:337:a2002.

7. Watanabe Y, Saito I, Henmi I, Yoshimura K, Maruyama K, Yamauchi K, et al. Skipping breakfast is correlated with obesity. J Rural Med. 2014;9:51-8.

8. Geliebter A, Astbury NM, Aviram-Friedman R, Yahav E, Hashim S. Skipping breakfast leads to weight loss but also elevated cholesterol compared with consuming daily breakfasts of oat porridge or frosted cornflakes in overweight individuals: a randomized controlled trial. J Nutr Sci. 2014;3:e56.

9. Odegaard AO, Jacobs DR Jr, Steffen LM, Van Horn L, Ludwig DS, Pereira MA. Breakfast frequency and development of metabolic risk. Diabetes Care. 2013;36(10):3100-6.

10. Baron KG, Reid KJ, Kern AS, Zee PC. Role of sleep timing in caloric intake and BMI. Obesity. 2011;19:1374-81.

11. Berg C, Lappas G, Wolk A, Strandhagen E, Toren K, Rosengren A, et al. Eating patterns and portion size associated with obesity in a Swedish population. Appetite. 2009;52:21-6. 
12. Wang JB, Patterson RE, Ang A, Emond JA, Shetty N, Arab L. Timing of energy intake during the day is associated with the risk of obesity in adults. J Hum Nutr Diet. 2014;27(suppl 2):255-62.

13. Soga $Y$, Shirai $C$, ljichi A. Association between daily lifestyle and the risk of metabolic syndrome among young adults in Japan. An analysis of Kobe city young adult health examination data. Nihon Koshu Eisei Zasshi. 2013;60:98-106

14. Kutsuma A, Nakajima K, Suwa K. Potential association between breakfast skipping and concomitant late-night-dinner eating with metabolic syndrome and proteinuria in the Japanese population. Scientifica (Cairo). 2014;2014:253581.

15. McHill AW, Melanson EL, Higgins J, Connick E, Moehlman TM, Stothard ER, et al. Impact of circadian misalignment on energy metabolism during simulated nightshift work. Proc Natl Acad Sci. U S A. 2014;111: 17302-7.

16. Ashizawa E, Katano S, Harada A, Yanagibori R, Kobayashi Y, Sato S, et al. Exploring the between standard lifestyle questionnaires administered during specific medical check-ups and incidence of metabolic syndrome in Chiba prefecture. Nihon Koshu Eisei Zasshi. 2014;61:176-85.

17. Overview of Estimates of National Medical Care Expenditure, FY2016. http:// www.mhlw.go.jp/toukei/saikin/hw/k-iryohi/16/index.html. Accessed 20 Oct 2018.

18. Body mass index - BMI. www.euro.who.int/en/health-topics/diseaseprevention/nutrition/a-healthy-lifestyle/body-mass-index-bmi. Accessed 20 Oct 2018.

19. Ministry of Health, Labour and Welfare. Standardized health checkup and intervention program (revised edition). 2013. http://www.mhlw.go.jp/ seisakunitsuite/bunya/kenkou_iryou/kenkou/seikatsu/dl/hoken-program1. pdf. Accessed 15 Jan 2018

20. International Diabetes Federation. IDF Consensus Worldwide Definition of the Metabolic Syndrome. 2006. https://www.idf.org/e-library/ consensus-statements/60-idfconsensus-worldwide-definitionof-themetabolic-syndrome. Accessed 20 Oct 2018.

21. National Health and Nutrition Examination Survey. https://www.mhlw.go.jp/ bunya/kenkou/kenkou_eiyou_chousa.html. Accessed 16 Aug 2018.

22. Tajima M, Lee JS, Watanabe E, Park JS, Tsuchiya R, Fukahori A, et al. Association between changes in 12 lifestyle behaviors and the development of metabolic syndrome during 1 year among workers in the Tokyo metropolitan area. Circ J. 2014;78:1152-9.

23. Assmann SF, Hosmer DW, Lemeshow S, Mundt KA. Confidence intervals for measures of interaction. Epidemiology. 1996;7:286-90.

24. Burgoine T, Forouhi NG, Griffin SJ, Brage S, Wareham NJ, Monsivais P. Does neighborhood fast-food outlet exposure amplify inequalities in diet and obesity? A cross-sectional study. Am J Clin Nutr. 2016;103:1540-7.

25. Knol MJ, VanderWeele TJ. Recommendations for presenting analyses of effect modification and interaction. Int J Epidemiol. 2012;41:514-20.

26. Hibi M, Masumoto A, Naito Y, Kiuchi K, Yoshimoto Y, Matsumoto M, et al. Night time snacking reduces whole body fat oxidation and increases LDL cholesterol in healthy young women. Am J Physiol Regul Integr Comp Physiol. 2012;304:R94-R101

27. Balieiro LC, Rossato LT, Waterhouse J, Paim SL, Mota MC, Crispim CA. Nutritional status and eating habits $s$ of bus drivers during the day and night. Chronobiol Int. 2014;31:1123-9.

28. Nakajima K, Suwa K. Association of hyperglycemia in a general Japanese population with late-night-dinner eating alone, but not breakfast skipping alone. J Diabetes Metab Disord. 2015;14:16.

29. Cahill LE, Chiuve SE, Mekary RA, Jensen MK, Flint AJ, Hu FB, et al. Prospective study of breakfast eating and incident coronary heart disease in a cohort of male US health professionals. Circulation. 2013;128:337-43.

30. Gallant AR, Lundgren J, Drapeau V. The night-eating syndrome and obesity. Obes Rev. 2012;13(6):528-36.

31. Garaulet M, Stanchez-Moreno C, Smith CE, Lee YC, Nicolas F, Ordovas JM. Ghrelin, sleep reduction and evening preference: relationships to CLOCK 3111 T/C SNP and weight loss. PLoS One. 2011;6:e17435.

32. Bo S, Fadda M, Castiglione A, Ciccone G, De Francesco A, Fedele D, et al. Is the timing of caloric intake associated with variation in diet-induced thermogenesis and in the metabolic pattern? A randomized crossove study. Int J Obes. 2015;39:1689-95.

33. Ribeiro DCO, Hampton SM, Morgan L, Deacon S, Arendt J. Altered postprandial hormone and metabolic responses in a simulated shift work environment. J Endocrinol. 1998;158:305-10.
34. Kato H, Kuninobu K, Saito A, Ideguchi K, Nishida Y, Kato H. Chrononutrition and health promotion. Nihon Yakurigaku Zasshi. 2011;137:120-4 In Japanese.

35. Shimba S, Ishii N, Ohta Y, Ohno T, Watabe Y, Hayashi M, et al. Brain and muscle Arnt-like protein-1 (BMAL1), a component of the molecular clock, regulates adipogenesis. Proc Natl Acad Sci U S A. 2005;102:12071-6.

36. Cao H. Adipocytokines in obesity and metabolic disease. J Endocrinol. 2014; 220:T47-59.

37. Lund J, Arendt J, Hampton SM, English J, Morgan LM. Postprandial hormone and metabolic responses amongst shift workers in Antarctica. J Endocrinol. 2001;171:557-64

38. Nakamoto M, Sakai T, Shuto E, Nakano A, Kosugi C, Hata A, et al. Period between dinner and bedtime is related to hypertension. Nippon Eiyo Shokuryo Gakkaishi. 2013;66:185-93 In Japanese.

39. Calvin AD, Carter RE, Adachi T, Macedo PG, Albuquerque FN, Walt C, et al. Effects of experimental sleep restriction on caloric intake and activity energy expenditure. Chest. 2013;144:79-86.

40. Markwald RR, Melanson EL, Smith MR, Higgins J, Perreault L, Eckel RH, et al. Impact of insufficient sleep on total daily energy expenditure, food intake, and weight gain. Proc Natl Acad Sci U S A. 2013;110:5695-700.

41. Nedeltcheva AV, Kilkus JM, Imperial J, Kasza K, Schoeller DA, Penev PD. Sleep curtailment is accompanied by increased intake of calories from snacks. Am J Clin Nutr. 2009:89:126-33.

42. Spaeth AM, Dinges DF, Goel N. Effects of experimental sleep restriction on weight gain, caloric intake, and meal timing in healthy adults. Sleep. 2013; 36:981-90.

43. Taylor LE, Sullivan JC. Sex differences in obesity-induced hypertension and vascular dysfunction: a protective role for estrogen in adipose tissue inflammation? Am J Physiol Regul Integr Comp Physiol. 2016;311:R714-20.

44. Lee CG, Carr MC, Murdoch SJ, Mitchell E, Woods NF, Wener MH, et al. Adipokines, inflammation, and visceral adiposity across the menopausal transition: a prospective study. J Clin Endocrinol Metab. 2009;94:1104-10.

\section{Ready to submit your research? Choose BMC and benefit from:}

- fast, convenient online submission

- thorough peer review by experienced researchers in your field

- rapid publication on acceptance

- support for research data, including large and complex data types

- gold Open Access which fosters wider collaboration and increased citations

- maximum visibility for your research: over $100 \mathrm{M}$ website views per year

At BMC, research is always in progress.

Learn more biomedcentral.com/submissions 